\title{
Direct atom imaging by chemical-sensitive
}

\section{holography: Supplementary Information}

\author{
Tobias Lühr, ${ }^{*, \dagger}$ Aimo Winkelmann ${ }^{\ddagger}{ }^{\dagger}$ Gert Nolze, ${ }^{\top}$ Dominique Krull, ${ }^{\dagger}$ and Carsten \\ Westphal ${ }^{\dagger}$ \\ Experimental Physics I, TU Dortmund, Dortmund, Germany, Experimental Department I, \\ Max Planck Institute of Microstructure Physics, Halle, Germany, and Department 5 - \\ Materials Engineering, BAM - Federal Institute for Materials Research and Testing, \\ Berlin, Germany \\ E-mail: tobias.luehr@tu-dortmund.de
}

\section{Supplementary information}

As demonstrated in the main text, the holographic reconstruction of electron diffraction patterns leads to spatially and chemically resolved atom images. However, in order to investigate aspects like the limitation in resolution or the influence of the inevitable twin image, a more mathematical examination is required. In the following we will give a deeper insight in the reconstruction scheme by a theoretical investigation.

Unless it is explicitly mentioned, we assume coordinate systems with origin at the emitter location and a z-axis oriented perpendicular to the sample surface. Therefore, the absolute

${ }^{*}$ To whom correspondence should be addressed

†TU Dortmund

${ }^{\ddagger}$ Max Planck Institute of Microstructure Physics

ॠBAM - Federal Institute for Materials Research and Testing 
values of all position vectors automatically correspond to the emitter distance. The measured intensity of a diffraction pattern $I$ to electrons with wavenumber $k$ can be written as the absolute square of a superposition of the initial wave $\psi_{0}$ with all scattered waves $\psi_{\mu}$.

$$
I=\left|\psi_{0}+\sum_{\mu} \psi_{\mu}\right|^{2}
$$

Without limitation, the scattered wave of the $\mu$-th atom with location at $\mathbf{r}_{\mu}$ can be described as

$$
\psi_{\mu}=A_{\mu} \cdot e^{i\left(k\left|\mathbf{r}-\mathbf{r}_{\mu}\right|+\Delta \varphi_{\mu}\right)}
$$

since the atom's scatter characteristic and multiple scattering effects can efficiently be expressed by anisotropic real functions for amplitude $A_{\mu}=A_{\mu}(\Theta, \Phi)$ and phase shift $\Delta \varphi_{\mu}=$ $\Delta \varphi_{\mu}(\Theta, \Phi)$. The vector $\mathbf{r}$ corresponds to the location of a pattern point. Since the distance $r=|\mathbf{r}|$ between emitter and the diffraction pattern is significantly higher than the distance $r_{\mu}=\left|\mathbf{r}_{\mu}\right|$ between emitter and scatter atom, the wave phase in equation (2) can be approximated by

$$
k\left|\mathbf{r}-\mathbf{r}_{\mu}\right| \approx k r_{\mu}-\mathbf{k} \cdot \mathbf{r}_{\mu}, \text { with } \mathbf{k}=k\left(\begin{array}{c}
\sin (\Theta) \cos (\Phi) \\
\sin (\Theta) \sin (\Phi) \\
\cos (\Theta)
\end{array}\right)
$$

Inserting equations (2) and (3) into equation (1) results in the following expression for an electron diffraction pattern:

$$
\begin{aligned}
I=A_{0}^{2}+\sum_{\mu} A_{\mu}^{2}+2 A_{0} \sum_{\mu} A_{\mu} & \cos \left(k r_{\mu}-\mathbf{k} \cdot \mathbf{r}_{\mu}+\Delta \varphi_{\mu}\right) \\
& +2 \sum_{\mu<\nu} A_{\mu} A_{\nu} \cos \left(k \Delta r_{\mu \nu}-\mathbf{k} \cdot \mathbf{r}_{\mu \nu}+\Delta \varphi_{\mu \nu}\right)
\end{aligned}
$$


We applied the following substitutions in this equation: $\Delta r_{\mu \nu}:=\left|\mathbf{r}_{\mu}\right|-\left|\mathbf{r}_{\nu}\right|, \mathbf{r}_{\mu \nu}:=\mathbf{r}_{\mu}-\mathbf{r}_{\nu}$, and $\Delta \varphi_{\mu \nu}:=\Delta \varphi_{\mu}-\Delta \varphi_{\nu}$. For experimental datasets the first two terms can be removed by applying the so-called anisotropy function $\chi$.

$$
\chi(\mathbf{r})=I(\mathbf{r})-I_{0}, \text { with } I_{0}=\frac{1}{N} \sum_{j=1}^{j=N} I\left(\mathbf{r}_{j}\right)
$$

The average intensity $I_{0} \approx A_{0}^{2}+\sum_{j} A_{\mu}^{2}$ of measured intensity to various locations $\mathbf{r}_{j}$ corresponds approximately to the non-modulated terms in equation (4). Then, we inserted the remaining terms of equation (4) into the reconstruction integral of equation (1) in the main text. Since the sequence of integration and summation may be exchanged, the reconstruction integral can be applied to each remaining summand in equation (4) first. This allows a separated analysis of the imaging properties of individual atoms.

$$
\begin{array}{ll}
\Gamma_{k}(\mathbf{r})= & \sum_{\mu}\left[\Gamma_{\mu}^{+}(\mathbf{r})+\Gamma_{\mu}^{-}(\mathbf{r})\right]+\sum_{\nu<\mu}\left[\Gamma_{\mu \nu}^{+}(\mathbf{r})+\Gamma_{\mu \nu}^{-}(\mathbf{r})\right] \\
\text { with } \quad & \Gamma_{\mu}^{ \pm}(\mathbf{r}):=A_{0} A_{\mu} e^{ \pm i\left(k r_{\mu}+\Delta \varphi_{\mu}\right)} \widetilde{\Gamma}\left(\mathbf{r} \mp \mathbf{r}_{\mu}\right) \text { and } \\
& \Gamma_{\mu \nu}^{ \pm}(\mathbf{r}):=A_{\mu} A_{\nu} e^{ \pm i\left(k r_{\mu \nu}+\Delta \varphi_{\mu \nu}\right)} \widetilde{\Gamma}\left(\mathbf{r} \mp \mathbf{r}_{\mu \nu}\right) .
\end{array}
$$

The complete image function $\Gamma_{k}$ corresponds to a superposition of identical image functions $\widetilde{\Gamma}$ with

$$
\widetilde{\Gamma}(\widetilde{\mathbf{r}})=2 \pi \int_{0}^{\Theta_{\max }} J_{0}\left(k \sqrt{\widetilde{x}^{2}+\widetilde{y}^{2}} \cdot \sin (\Theta)\right) \sin (\Theta) e^{i k \widetilde{z} \cos (\Theta)} d \Theta
$$

Here, $J_{0}$ denotes the 0 th Bessel function of the first kind. In order to show the imaging properties of the $\widetilde{\Gamma}$-function, calculations of equation (9) were performed. Some of the results are displayed in figure S1. The spatial coordinates are expressed in multiples of the electron wavelength, in order to keep the illustration valid for a general case. Since the integrand of $\Gamma^{ \pm}$remains constant for $\sqrt{\widetilde{x}^{2}+\widetilde{y}^{2}}=$ const., the image function of a single atom is of rotational symmetry around the $\widetilde{z}$-axis. Hence, the plots in figure S1 contain all 
information about the imaging properties of $\widetilde{\Gamma}$. The image function $\widetilde{\Gamma}(\widetilde{\mathbf{r}})$ provides a global maximum for all $\Theta_{\max }$ at location $\widetilde{\mathbf{r}}=\mathbf{0}$. With increasing absolute value of $\widetilde{\mathbf{r}}$ the $\widetilde{\Gamma}$-function rapidly decreases. Therefore, the peaks in the final image function $\Gamma_{k}$ according to equation (6) are generated by the $\widetilde{\Gamma}$-functions of equations (7) and (8). Further, the maxima of the $\widetilde{\Gamma}$-functions become broader with decreasing opening-angle $\Theta_{\max }$ of the diffraction pattern, as displayed in figure S1. The peak broadening perpendicular to the $\widetilde{z}$-axis is very shallow even for small $\Theta_{\max }$, shown in figure S1a. In contrast, figure S1b clearly demonstrates an elongation along the $\widetilde{z}$-axis depending on the opening-angle. Now, in the final image function according to equation (6) the peak locations can be interpreted easily. For this the arguments of the $\widetilde{\Gamma}$-functions in equations (7) and (8) must be analysed. The $\Gamma_{\mu}^{ \pm}$in

.png
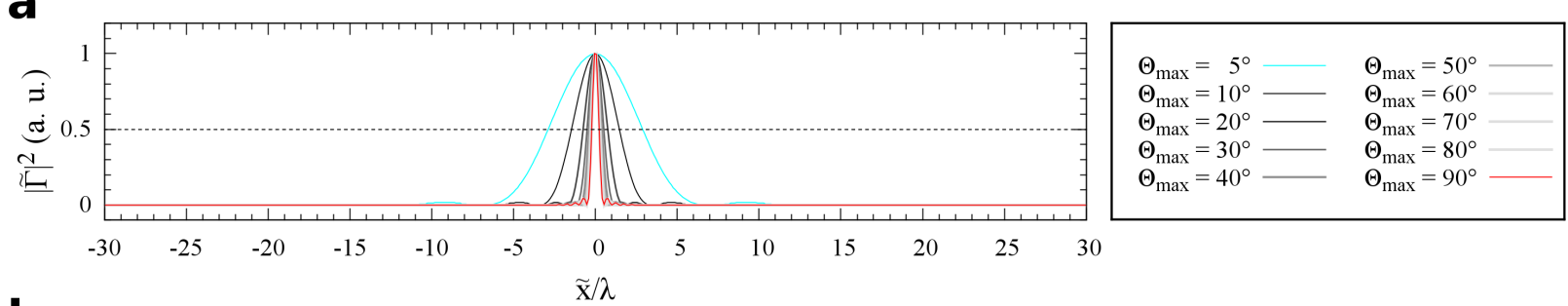

b
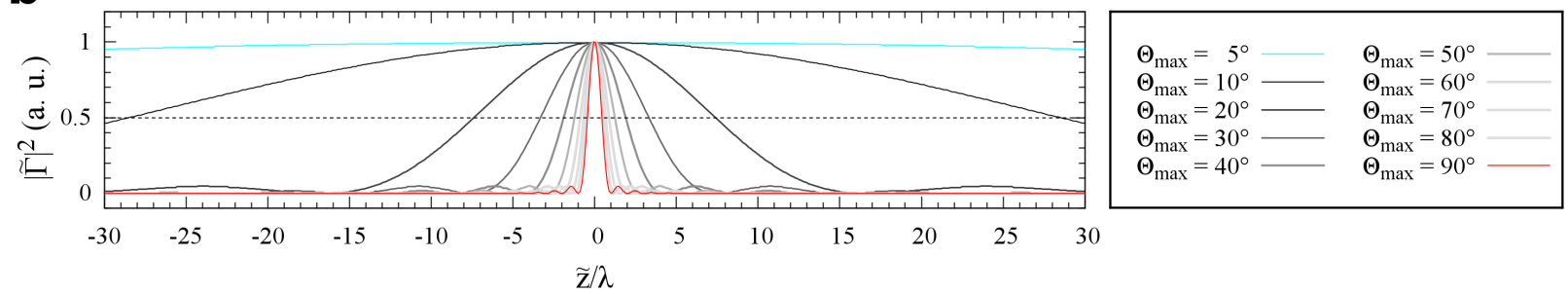

Figure S1: Calculation of the dependence of the $\widetilde{\Gamma}$-functions on the opening-angle $\Theta_{\max }$ of the corresponding hemispherical diffraction pattern. a, Absolute square of the $\widetilde{\Gamma}$-function perpendicular to the $\widetilde{z}$-axis. b, Absolute square of the $\widetilde{\Gamma}$-function along the $\widetilde{z}$-axis.

equation (7) represent peaks at the locations $\mp \mathbf{r}_{\mu}$. Hence, peaks of the $\Gamma_{\mu}^{+}$-functions reflect locations of the scatter atoms, while $\Gamma_{\mu}^{-}$-functions describe the pointsymmetric twin image. In equation (8) the peaks from the $\Gamma_{\mu \nu}^{ \pm}$-functions are located at $\mp \mathbf{r}_{\mu \nu}$. Since the vectors $\mp \mathbf{r}_{\mu \nu}$ point from the $\mu$-th scatter atom to the $\nu$-th scatter atom and vice versa, the $\Gamma_{\mu \nu}^{ \pm}$-functions correspond to an image superposition of all scatter atoms with their local environments. 
Fortunately, the amplitude $A_{0}$ of the emitter wave is always larger than the amplitudes $A_{\mu}$ of the scatter atoms. Therefore, the contribution of the structure relevant $\Gamma_{\mu}^{ \pm}$-functions to the complete image function is larger than the contribution of the $\Gamma_{\mu \nu}^{ \pm}$-functions, according to equation (7) and (8). Thus, we will focus the discussion on the $\Gamma_{\mu}^{ \pm}$-functions in the following.

In the main text, we investigated the diffraction patterns of electrons with kinetic energies of $E_{k i n} \geq 10 \mathrm{keV}$. Scattered waves at these energies are constrained within a cone in the forward direction. In a rough approximation the anisotropic amplitude and phase shift of a scattered wave can be described as

$$
A_{\mu}(\Theta, \Phi) e^{i \Delta \varphi_{\mu}(\Theta, \Phi)}=\left\{\begin{array}{cc}
A_{\mu} e^{i \Delta \varphi_{\mu}}=\text { const. } & \text { for } \measuredangle \mathbf{r}^{\prime}, \mathbf{r}_{\mu} \leq \beta_{\max } \\
0 & \text { e. }
\end{array}\right.
$$

In this equation $\beta_{\max }$ corresponds to the opening-angle of the scatter cone. Hence, the $\Gamma_{\mu}^{ \pm}$-functions according to equation (7) remain legit in a coordinate system with the $\mathrm{z}^{\prime}$ axis aligned along $\mathbf{r}_{\mu}$. Now, $\Theta_{\max }$ in equation (9) must be replaced by $\beta_{\max }$. Thus, the corresponding atom location is reconstructable via the reconstruction integral (1) from the main text.

While the $\Gamma_{\mu}^{+}$-peak indicates the true atom location at $+\mathbf{r}_{\mu}$, a second $\Gamma_{\mu}^{-}$-peak can be found at $-\mathbf{r}_{\mu}$, always. Generally, this twin image complicates the interpretation of reconstructions, since the $\Gamma_{\mu}^{+}$-peaks and $\Gamma_{\mu}^{-}$-peaks provide identical image intensities. Further, true and twin image may interfere destructively, especially for samples with high translational symmetry. Fortunately, electron scattering at high kinetic energy prevents a superposition of true and twin image. An atom appears in the reconstructed image only if its scattered wave leaves the sample. Due to the strong forward scattering, this condition is solely valid for those atoms being located between emitter and sample surface. In figure S2 the visible region is located between the red and blue line. All electrons being scattered below the emitter plane progate into the bulk. Thus, the sample region located below the emitter remains 
invisible in the reconstruction. Since the $\Gamma_{\mu}^{-}$-peaks correspond to an inversion of the $\Gamma_{\mu}^{+-}$ peaks through the emitter, true and twin image are spatially separated in regions located above and below the emitter, respectively. Therefore, both images automatically occur in the reconstruction without superposition. Figure S2 also shows another very important aspect

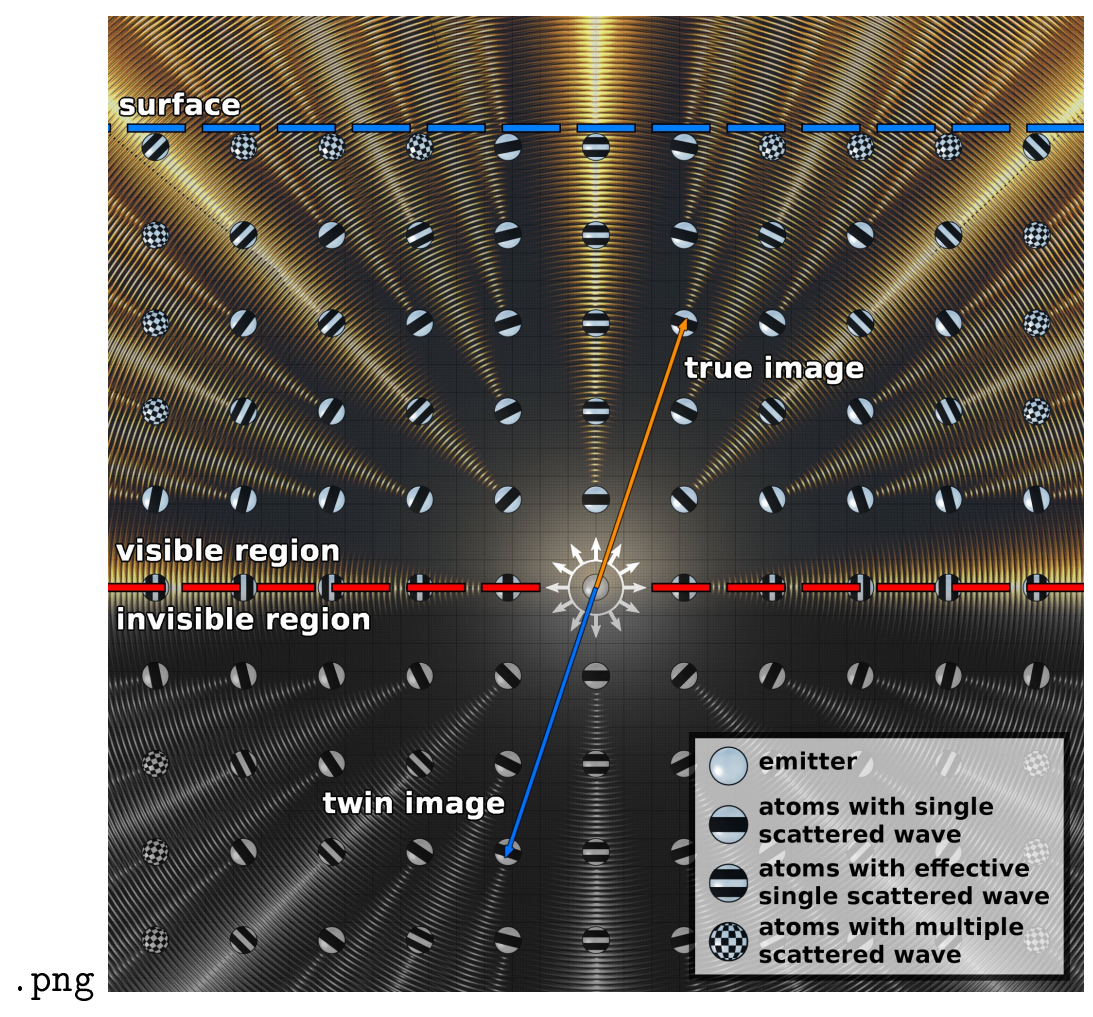

Figure S2: Scattered waves in the emitter environment. Only scattered waves of atoms above the emitter contribute to the diffraction pattern, since they will leave the sample finally, due to the strong forward scattering. The region below the emitter remains invisible in the reconstruction, which prevents twin image superposition. Additionally, atoms within a range of 1-2 $\mathrm{nm}$ provide scattered waves, with single and effective single scattered waves.

of the approximated wave model according equation (10). Generally, electrons exhibit a high scatter probability leading to multiple scattering effects. As visualised in figure S2, multiple scattering can occur in a certain distance to the emitter only. Scattered waves of atoms located very close to the emitter can be generated by the emitter wave only, due to strong forward scattering. Therefore, these atoms are sources of single scattered waves. Further, multiple scattered waves resulting from several waves, and which come from the 
same direction, may be interpreted as an effective single scattered wave. This situation occurs for atoms which are arranged along a line with the emitter and other scatter atoms. In figure S2 almost all atoms generate either single scattered waves or effective single scattered waves. Therefore, multiple scattering will not affect the reconstruction within a range of approximately 1-2 $\mathrm{nm}$. This estimate is based on the opening-angle $\beta_{\max }$ of the scatter cone and on the investigated sample structure.

In the main text, the difference $\chi_{k_{2}}-\chi_{k_{1}}$ of two electron diffraction patterns with the wavenumbers $k_{1}$ and $k_{2}$ was applied in the reconstruction integral according to equation (1) from the main text. For all reconstructions the average wavenumber $\bar{k}=\frac{1}{2}\left(k_{1}+k_{2}\right)$ was used. ${ }^{1}$ In a coordinate system with the $\mu$-th scatter atom on the $\mathrm{z}^{\prime}$-axis the corresponding structure relevant $\widetilde{\Gamma}_{\mu}^{ \pm}$-functions can be calculated to

$$
\begin{aligned}
\widetilde{\Gamma}_{\mu}^{ \pm}(\mathbf{r})=2 \pi i A_{0} A_{\mu} e^{ \pm i\left(\bar{k} r_{\mu}+\Delta \varphi_{\mu}\right)} \int_{0}^{\beta_{\max }} J_{0}\left(\bar{k} \sqrt{x^{\prime 2}+y^{\prime 2}} \cdot \sin \left(\Theta^{\prime}\right)\right) \sin \left(\Theta^{\prime}\right) \times \\
\sin \left(\delta k r_{\mu}\left(1-\cos \left(\Theta^{\prime}\right)\right)\right) e^{i \bar{k}\left(z^{\prime} \mp r_{\mu}\right) \cos \left(\Theta^{\prime}\right)} d \Theta^{\prime} .
\end{aligned}
$$

The difference of the wavenumbers was substituted by $2 \delta k:=k_{2}-k_{1}$. In comparison, in equation (9) the integrand provides an additional factor $\sin \left(\delta k r_{\mu}\left(1-\cos \left(\Theta^{\prime}\right)\right)\right)$. Calculations to equation (11) showed that the $\widetilde{\Gamma}_{\mu}^{+}$-function according to equation (11) provide almost identical curves perpendicular to the $\mathrm{z}^{\prime}$-axis, as shown in figure S1. However, the $\widetilde{\Gamma}_{\mu}^{ \pm}$-function may differ along $\mathrm{z}^{\prime}$-axis depending on the values for $\delta k, r_{\mu}$, and $\beta_{\max }$. Figure S3a displays the $\widetilde{\Gamma}_{\mu}^{ \pm}$-function at different values for $\delta k$. At the smallest $\delta k$ the function $\widetilde{\Gamma}_{\mu}^{ \pm}$provides a single global maximum. With increasing $\delta k$ the function $\widetilde{\Gamma}_{\mu}^{ \pm}$splits into two peaks. In order to avoid the peak-splitting in the image function, the energy difference between the two diffraction patterns must be as small as possible. Additionally, the image peaks obtained from the difference pattern split if the distance between emitter and scatter atom becomes too large, as visualised in figure S3b. Thus, single image peaks indicating atom locations can only be found within a limited emitter-scatter atom distance. Figure S3c shows the influence 
of the scatter cone opening-angle on the image peak splitting. In figure S3, the curves with a setting-in of the peak-splitting are indicated in red. From comparing the parameters which enter in the peakform of the $\widetilde{\Gamma}_{\mu}^{+}$-function we find:

$$
\delta k r_{\mu}\left(1-\cos \left(\beta_{\max }\right)\right) \approx 1.3 \pi
$$

This condition is an estimate of the region, where atom locations are indicated by a single intensity peak. Atom images can be found without splitting for an emitter distance up to several nanometers, as clearly demonstrated by the $\widetilde{\Gamma}_{\mu}^{ \pm}$-curves in figure $\mathrm{S} 3$. In the main text, this finding is confirmed by all reconstructions.

The final aspect in our discussion adresses efficient noise and artefact suppression in equation
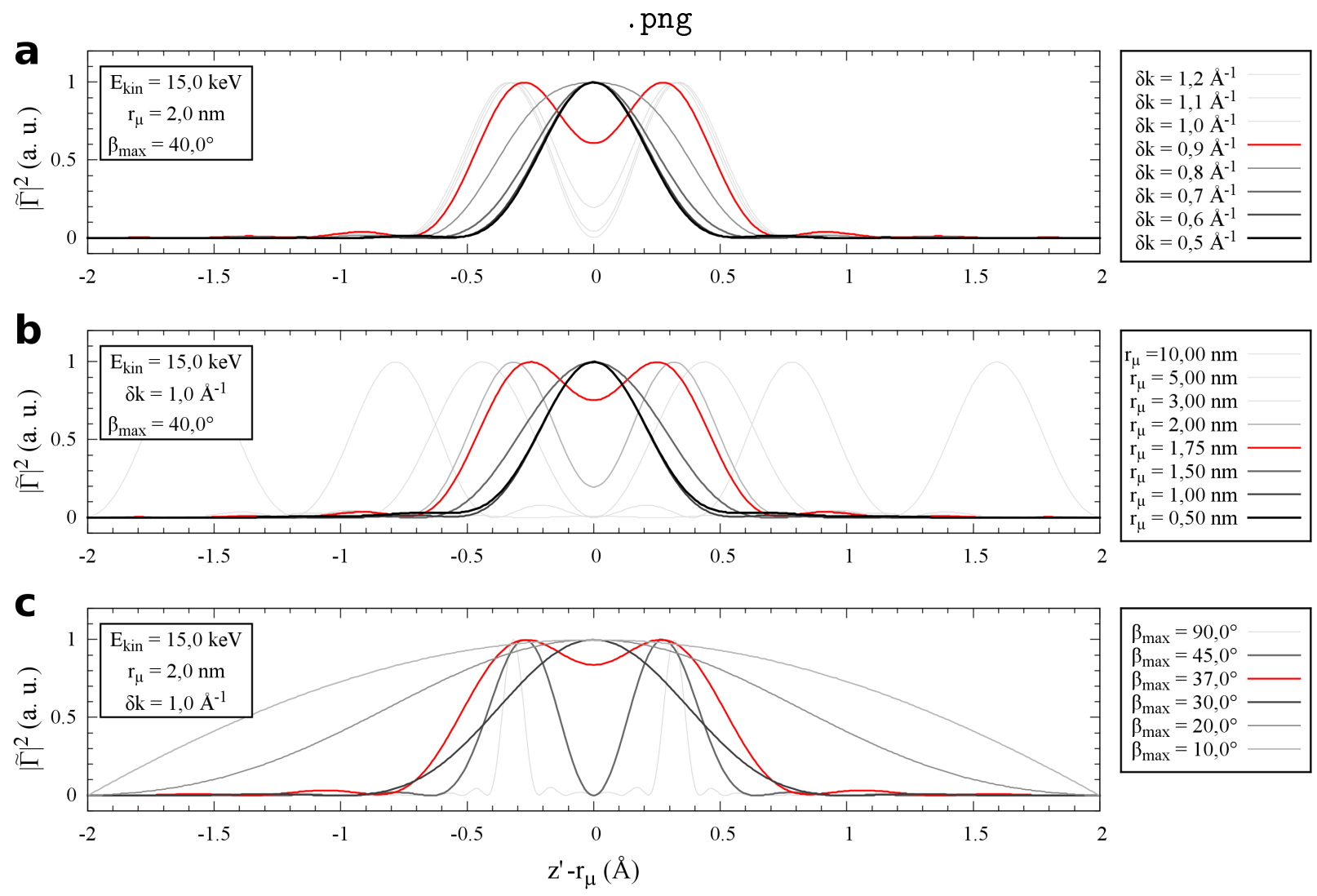

Figure S3: Peak function $\widetilde{\Gamma}_{\mu}^{ \pm}$of the difference pattern. a, Peak function at several wavenumber differences $\delta k$ between the individual diffraction patterns. $\mathrm{b}$, Peaks of the $\widetilde{\Gamma}_{\mu}^{ \pm}$-function for various emitter-scatter atom distances. $c$, Influence of the scatter cone opening-angle on the peak function $\widetilde{\Gamma}_{\mu}^{ \pm}$. 
(3) of the main text. Due to translational symmetry of the samples, the reconstructed images are invariant to shifts along the lattice vectors $\mathbf{G}_{\text {uvw }}$. In order to avoid destructive interferences between the complex image functions, we inserted the factor $e^{-i \bar{k}\left|\mathbf{r}+\mathbf{G}_{u v w}\right|}$ in the equation (3) from the main text. At atom locations $\mathbf{r}_{\mu}$ the integral in the peak function $\widetilde{\Gamma}_{\mu}^{+}\left(\mathbf{r}_{\mu}\right)$ of equation (11) is real always. Therefore, the complex phase of the $\mu$-th scatter atom's peak function is solely determined by the factor $C_{\mathbf{r}}^{+}$outside the integral.

$$
C_{\mathbf{r}}^{+}:=2 \pi i A_{0} A_{\mu} e^{+i\left(\bar{k} r+\Delta \varphi_{\mu}\right)}
$$

According to this equation, the shifted function $\widetilde{\Gamma}_{\mu}^{+}\left(\mathbf{r}_{\mu}+\mathbf{G}_{u v w}\right)$ at the atom location $\mathbf{r}_{\mu}$ provides a phase value of $\bar{k}\left|\mathbf{r}_{\mu}+\mathbf{G}_{u v w}\right|+\Delta \varphi_{\mu}$. Multiplying the shifted image functions with the factor $e^{-i \bar{k}\left|\mathbf{r}+\mathbf{G}_{u v w}\right|}$ is yielding at atom location $\mathbf{r}_{\mu}$ :

$$
e^{-i \bar{k}\left|\mathbf{r}+\mathbf{G}_{u v w}\right|} \cdot C_{\mathbf{r}_{\mu}+\mathbf{G}_{u v w}}^{+}=2 \pi i A_{0} A_{\mu} e^{i \Delta \varphi_{\mu}}
$$

Now, in a shifted image function the phase of the $\Gamma^{+}$-peaks becomes independent from the shift $G_{u v w}$. Additionally, the phase is not depending on the wavenumber $\bar{k}$. Hence, a superposition of several shifted image functions accumulates peak maxima, while the modulations around the peaks average out. Therefore, it is impossible to randomly generate peaks at locations $\mathbf{r}_{\mu} \neq \mathbf{G}_{\text {uvw }}$ that are not related to atom locations by applying equation (3) from the main text.

As mentioned in the main text, the lattice vectors $\mathbf{G}_{u v w}$ can easily be identified by searching for the strongest intensity peaks in the native reconstruction resulting from equation (1) in the main text. Thus, no ab-initio information is needed for applying equation (3) from the main text. As a nice sideeffect, the $\Gamma_{\mu}^{-}$-peaks as well as the $\Gamma_{\mu \nu}^{ \pm}$-functions still depend on $k$, after multiplication by $e^{-i \bar{k}\left|\mathbf{r}+\mathbf{G}_{u v w}\right|}$. With an increasing number of difference patterns obtained at different energies, these peaks will average out. Therefore, only $\Gamma_{\mu}^{+}$-maxima indicating true atom locations will remain in the reconstructed image. 
Summarising the discussion, we presented an approach explaining the imaging properties of electron diffraction patterns by means of a holographic reconstruction. At kinetic energies of $E_{k i n} \geq 10 \mathrm{keV}$ scattered electron waves generate intensity modulations that can be reconstructed by applying equation (1) from the main text. A superposition of true and twin image is prevented, due to the strong forward scattering. Further, it was demonstrated that a difference pattern allows a successful reconstruction retrieving atom locations in the emitter environment. The lattice points $\mathbf{G}_{u v w}$, which are indicated by the strongest reconstructed peaks in a native reconstruction, can be used for an efficient artifact and noise reduction.

\section{References}

(1) Omori, S.; Nihei, Y.; Rotenberg, E.; Denlinger, J. D.; Marchesini, S.; Kevan, S. D.; Tonner, B. P.; Van Hove, M. A.; Fadley, C. S. Phys. Rev. Lett. 2002, 88, 055504. 